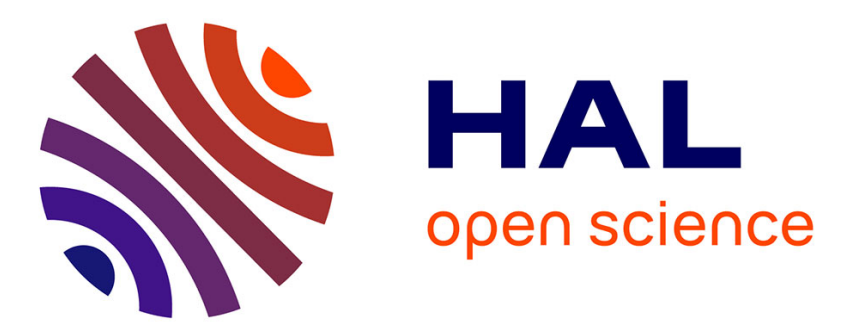

\title{
A 3D pseudospectral method for cylindrical coordinates. Application to the simulations of rotating cavity flows
}

\author{
Noele Peres, Sébastien Poncet, Eric Serre
}

\section{To cite this version:}

Noele Peres, Sébastien Poncet, Eric Serre. A 3D pseudospectral method for cylindrical coordinates. Application to the simulations of rotating cavity flows. Journal of Computational Physics, 2012, 231, pp.6290-6305. 10.1016/j.jcp.2012.04.033 . hal-00822026

\section{HAL Id: hal-00822026 \\ https://hal.science/hal-00822026}

Submitted on 13 May 2013

HAL is a multi-disciplinary open access archive for the deposit and dissemination of scientific research documents, whether they are published or not. The documents may come from teaching and research institutions in France or abroad, or from public or private research centers.
L'archive ouverte pluridisciplinaire HAL, est destinée au dépôt et à la diffusion de documents scientifiques de niveau recherche, publiés ou non, émanant des établissements d'enseignement et de recherche français ou étrangers, des laboratoires publics ou privés. 


\title{
A 3D pseudospectral method for cylindrical coordinates. Application to the simulations of rotating cavity flows
}

\author{
Noele PERES, Sébastien PONCET, Eric SERRE* \\ Laboratoire de Mécanique, Modélisation et Procédés Propres (M2P2), \\ UMR 7340 CNRS - Aix-Marseille Université - Ecole Centrale, \\ IMT La Jetée, Technopôle de Château Gombert, 38 rue F. Joliot Curie 13451 Marseille, \\ France
}

\begin{abstract}
The present work proposes a collocation spectral method for solving the three-dimensional Navier-Stokes equations using cylindrical coordinates. The whole diameter $-R \leq r \leq R$ is discretized with an even number of radial Gauss-Lobatto collocation points and an angular shift is introduced in the Fourier transform that avoid pole and parity conditions usually required. The method keeps the spectral convergence that reduces the number of grid points with respect to lower-order numerical methods. The grid-points distribution densifies the mesh only near the boundaries that makes the algorithm wellsuited to simulate rotating cavity flows where thin layers develop along the walls. Comparisons with reliable experimental and numerical results of the literature show good quantitative agreements for flows driven by rotating discs in tall cylinders and thin inter-disc cavities. Associated to a Spectral Vanishing Viscosity [E. Séverac and E. Serre J. Comp. Phys. 226 (2007) 1234-1255], the method provides very promising LES results of turbulent cavity flows.
\end{abstract}

Keywords: Cylindrical coordinates, pseudospectral method, collocation method, rotor-stator flow

\footnotetext{
*Corresponding author. Tel.: +334 911185 35; Fax: +334 91118502

Email address: eric.serre@L3m.univ-mrs.fr (Eric SERRE)
} 


\section{Introduction}

Cylindrical coordinate system is well-adapted to solve many physical configurations, including meteorological and geophysical problems, as well as ba-

sic fluid flows such as rotating cavity flows, pipe flows or jets. High-accurate numerical methods like spectral methods that are much less diffusive and dispersive than low order ones are highly desirable to investigate instability mechanisms and turbulent flows that require accuracy and long time integrations. However, dedicated numerical techniques must be developped to handle problems near the coordinate origin such as the singularity of the equations or the time-step limitation due to the clustering of mesh points.

Singularity is due to the presence of terms $1 / r^{n}(n=1,2)$ in the NavierStokes equations governing the flow, where $r$ is the radial distance, although the flow field itself does not exhibit any singular behaviour on the axis. Many studies in the literature have considered the special behaviour of flow equations at the origin of cylindrical coordinate systems and have proposed different techniques. Apparently these approaches depend greatly on whether a spectral, finite-volume or finite-difference method is used but in fact there are some similarities between them.

Methods using series expansions of the variables around singularity have been developed and applied using finite differences as proposed for example by Constantinescu and Lele [1]. These authors reinterpret the regularity conditions developed in the context of pseudospectral methods to develop a generally applicable numerical method for treating the singularity, when non axisymmetric flows are solved in cylindrical coordinates using highly accurate finite-difference schemes on non-staggered grids. Cartesian coordinate systems can be also considered by rewritten the equations to obtain a form similar to that in Cartesian coordinates [2]. Shifting the grid points in the radial direction by half the radial mesh spacing has been also proposed by Mohseni and Colonius [3].

In the context of spectral methods, Boyd [4] listed several options of radial basis sets and radial grids for a finite cylindrical domain. The first option is the use of Bessel functions. Huang and Sloan [5] used the eigenvalue problem of Bessel and Poisson equations on the unit disc to construct a pole condition from a smoothness assumption and the differential equation itself. Unfortunately, Bessel functions converge only at an algebraic rate. Orszag and Patera [6] employed Robert-type basis $r^{m} T_{n}(r)(m$ denotes the 
angular wavenumber and has the same parity as $n$ ) in polar coordinates to remove the singularity of the cylindrical system. The main disadvantage of this alternative option is that polar Robert functions are ill-conditioned and so can be used only at very low resolution. Another option listed by Boyd [4] is the use of one-sided Jacobi polynomials. The Fourier components $u_{m}(r, z)$ of any scalar function $u(r, \theta, z)$ of the cylindrical coordinates $(r, \theta, z)$ must satisfy the following condition: $u_{m}(r, z)=r^{|m|} U_{m}\left(r^{2}, z\right)$, with $U_{m}$ a regular function of the radial $r$ and axial $z$ coordinates. The first well-conditioned basis satisfying these regularity conditions and providing spectral accuracy has been proposed by Matsushima and Marcus [7] in the case of 2D Neumann boundary value problems and was based on one-sided Jacobi polynomials multiplied by $r^{m}$ for the radial coordinate. Zhang et al. [8] combined Jacobi polynomials as the expansion basis in the radial direction close to the axis and Legendre-Lagrangian interpolants away, and recast the governing equations in the spectral space so that the singularities were removed. Recently, Auteri and Quartapelle [9] developed solvers for the 3D Helmholtz equation in a finite cylinder based on Fourier series expansion in the tangential direction and Legendre polynomials in the axial direction. To overcome the pole singularity, they proposed a new Jacobi polynomial basis. Shen [10] proposed spectral-Galerkin methods to solve the Helmholtz equation written in cylindrical coordinates. As a solution to remove the singularity at the axis, all the velocity components are set to zero as pole conditions. Lopez and Shen [11] simulated the axisymmetric flow in a cylinder using a spectral-Galerkin method. They proposed on the axis a singular boundary condition, which is approximated by using a smooth boundary condition. In the physical space, it corresponds to set all variables to 0 at $r=0$. In the $3 \mathrm{D}$ case, the pressure requires to be single-valued at the pole [12]. In pipe flows, O'Sullivan and Breuer [13] avoided the singularity by using information in spectral space related to the series expansions of the velocity and pressure at $r=0$ and by increasing the resolution near the axis. Serre and Pulicani [14] proposed, in the context of collocation methods, a Chebyshev expansion between 0 and $\mathrm{R}$ (the radius of the cavity) and to avoid the polar problem, they introduced a change of dependent variables: $\mathbf{V}=r^{\beta} \mathbf{V}^{*}$ and $P=r^{\beta} P^{*}$, with $\beta<0$, which yields the conditions $u^{*}=v^{*}=w^{*}=p^{*}=0$ on the axis. The same idea was developed by Chen et al. [15] to solve the Poisson equation in cylindrical geometries. They multiplied the equation by a factor $r^{2}$ and made an adequate change of variables. Another way to avoid the pole problem when using spectral methods is to apply Gauss-Radau nodes as was done by Mil- 
lour and Labrosse [16] for solving the binary liquid thermal convection in a cylinder but applying axisymmetric boundary conditions on the axis.

Mercader et al. [17] proposed recently a spectral code used to study the Rayleigh-Bénard convection in a vertical stationary or rotating cylinder. They considered radial expansions along the diameter of the cell in order to avoid clustering around the axis and chose a selected number of points to ensure that the origin is not a collocation point. Moreover, they imposed implicitly some regularity conditions at $r=0$ by forcing the proper parity of the Fourier expansions in the radial direction since $(r, \theta+\pi, z)$ represents the same point as $(-r, \theta, z)$. The main advantage of their method is that it forces the minimal number of regularity conditions and that it reduces the size of the matrices.

The present work extends the collocation Chebyshev Fourier-Garlekin discretization scheme designed for annular computational domains in Raspo et al. [18] and that have proved to be highly accurate and computationally efficient for simulating instability patterns and turbulent flows in many annular rotating cavities (see some examples in [19]). The three-dimensional Navier-Stokes equations in cylindrical coordinates are discretized over the domain $[0,2 \pi] \times[-R, R] \times[0, H]$, with $\mathrm{R}$ the cavity radius and $\mathrm{H}$ the axial spacing of the cavity. An even number of collocation points in the radial direction avoids any pole condition at $r=0$. A non-homogeneous discretization is introduced in the angular direction through an angular shift equal to $\pi / 2 K$ ( $K$ the number of mesh points in that direction) for $\theta>\pi$ that avoids the use of parity conditions.

The paper is organized as follows. The Navier-Stokes equations written in cylindrical coordinates are first given in Section 2. The numerical approximation is presented in Section 3 and the validation of the method is checked in Section 4. The spectral convergence of the algorithm is shown using analytical solutions and its accuracy is assessed by comparisons with reliable results of the literature in rotor-stator cavity flows. Finally, Section 5 shows the ability of the method to perform LES of turbulent flows at Reynolds numbers $O\left(10^{5}\right)$ before concluding in Section 6 .

\section{Mathematical modelling}

An incompressible fluid flows within a cylindrical cavity of radius $R$ and height $H$. Its motion is governed by the three-dimensional Navier-Stokes 
equations written in the velocity-pressure formulation, together with the continuity equation and appropriate boundary conditions. The components of the velocity vector $\mathbf{V}$ are denoted $u, v$ and $w$ in the cylindrical coordinate system $(r, \theta, z)$ and $P$ is the pressure. The momentum and continuity equations, in dimensionless form can be written in the domain $D$ of border $\partial D$ :

$$
\frac{\partial \mathbf{V}}{\partial t}+(\mathbf{V} \cdot \nabla) \mathbf{V}=-\nabla P+\frac{1}{R e} \Delta \mathbf{V}+\mathbf{F} \text { in } \mathrm{D}
$$

$$
\nabla \cdot \mathbf{V}=0 \text { in } \mathrm{D}
$$

where $t$ is time and $\mathbf{F}$ represents a given body force.

Appropriate boundary conditions of Dirichlet type for the velocity vector write in a general way:

$$
\mathbf{V}=\mathbf{W} \text { on } \partial \mathrm{D}
$$

$\Delta$ is the vectorial Laplacian operator written for cylindrical coordinates:

$$
\begin{array}{r}
(\Delta V)_{r}=\nabla^{2} u-\frac{u}{r^{2}}-\frac{2}{r^{2}} \frac{\partial v}{\partial \theta} \\
(\Delta V)_{\theta}=\nabla^{2} v-\frac{v}{r^{2}}+\frac{2}{r^{2}} \frac{\partial u}{\partial \theta} \\
(\Delta V)_{z}=\nabla^{2} w \\
\nabla^{2}=\frac{\partial^{2}}{\partial r^{2}}+\frac{1}{r} \frac{\partial}{\partial r}+\frac{1}{r^{2}} \frac{\partial^{2}}{\partial \theta}+\frac{\partial^{2}}{\partial z^{2}}
\end{array}
$$

\section{Numerical approximation}

The resolution of Equations (1-3) leads to a Stokes problem coupling the velocity and the pressure. Collocation method requires boundary conditions in the physical space that can lead to an indetermination at $r=0$ when the domain is discretized over the radius for $r \in[0, R]$ as in [14]. 


\subsection{The $r=0$-singularity}

To avoid problems arising near the coordinate origin, the computational domain is discretized in the radial direction over the whole diameter between $-R$ and $R$ with an even number $N$ of Gauss-Lobatto collocation points, $r_{i}=\cos (i \pi / N), 0 \leq i \leq N$. Thus, no collocation point is located at $r=0$ and no pole condition is required. The discretization over the diameter leads to an overlapping of points in the azimuthal direction that usually requires parity conditions for all variables $\Psi$ such as $\Psi(r, \theta, z)=\Psi(-r, \theta+\pi, z)$.

Here, for a given even number of points $K$, a shift equal to $\pi /(2 K)$ for $\theta>\pi$ is introduced in the angular discretization over $[0,2 \pi]$, as formerly proposed by Heinrichs [20] to solve two-dimensional Poisson equations on an unit disc. According to the angular shift, the following discrete angles $\theta_{j}$ are defined by:

$$
\theta_{j}= \begin{cases}(j-1) \frac{2 \pi}{K} & \text { if } \quad j=1, \ldots, \frac{K}{2}, \\ \pi+\frac{\pi}{K}+\left(j-\left(\frac{K}{2}+1\right)\right) \frac{2 \pi}{K} & \text { if } \quad j=\frac{K}{2}+1, \ldots, K .\end{cases}
$$

An example is shown on Figure 1(a) for $K=8$. Such discretization clearly leads to a better resolution in the azimuthal direction with twice the amount of points although the number of Fourier modes remains equal to $K / 2$. An example of the 3D grid is shown on Figure 1(b). The distribution of GaussLobatto collocation points over the diameter tightens the points within the boundary layers along the walls and avoids unnecessary clustering of points around the axis.

According to this angular discretization, the following real Fourier basis can be introduced:

$$
\Phi_{k}(\theta)= \begin{cases}\sin (\mathrm{k} \theta) & \text { if } \quad k=1, \ldots, \frac{K}{2}-1, \\ \cos (\mathrm{k} \theta)+\sin (\mathrm{k} \theta) & \text { if } \quad k=\frac{K}{2}, \\ \cos \left(\left(\mathrm{k}-\left(\frac{\mathrm{K}}{2}+1\right)\right) \theta\right) & \text { if } \quad k=\frac{K}{2}+1, \ldots, K .\end{cases}
$$

that corresponds to the following $K \times K$ transformation matrix in spectral space:

$$
P_{\theta}=\left(\Phi_{k}\left(\theta_{j}\right)\right), \quad j, k=1, \ldots, K
$$




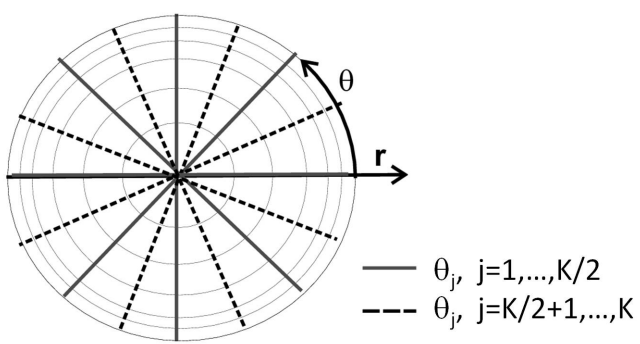

(a)

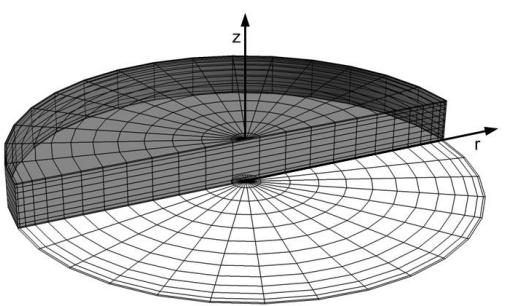

(b)

Figure 1: Grid structure: (a) Discrete angle $\theta_{j}$ distribution defined by Eq. (8) in a $(\theta, z)$ plane showing the angular shift. Black and dashed diameters correspond to discretization indices $j=1, \ldots, \frac{K}{2}$ and $j=\frac{K}{2}+1, \ldots, K$, respectively. Grey circles show the radial mesh distribution; (b) Example of 3D mesh discretization in a thin inter-disc cavity.

According to this basis, calculations of the first and second-order derivative operators in the azimuthal direction are straightforward both in physical and spectral spaces. The first derivation of any variable is given by the matrix product $D_{\theta} P_{\theta}^{-1} \Psi$, where $D_{\theta}$ is the following $K \times K$ full matrix:

$$
D_{\theta}=\left(\Phi_{k}^{\prime}\left(\theta_{j}\right)\right)_{1 \leq j, k \leq K}
$$

and for $1 \leq j \leq K$ :

$$
\Phi_{k}^{\prime}\left(\theta_{j}\right)=\left\{\begin{array}{lc}
k \cos \left(\mathrm{k} \theta_{\mathrm{j}}\right), & \mathrm{k}=1, \ldots, \frac{\mathrm{K}}{2}-1, \\
-k \sin \left(\mathrm{k} \theta_{\mathrm{j}}\right)+\mathrm{k} \cos \left(\mathrm{k} \theta_{\mathrm{j}}\right), & \mathrm{k}=\frac{\mathrm{K}}{2}, \\
-\left(k-\left(\frac{K}{2}+1\right)\right) \sin \left(\left(\mathrm{k}-\left(\frac{\mathrm{K}}{2}+1\right)\right) \theta_{\mathrm{j}}\right), & \mathrm{k}=\frac{\mathrm{K}}{2}+1, \ldots, \mathrm{K} .
\end{array}\right.
$$

As referred by Peyret [21], numerical experiments have shown that the presence of the term at $k=K / 2$ may lead to instabilities in time-dependent problems mainly because the first derivative cannot be represented in this basis. Therefore the mode $K / 2$ must be filtered. The second-order derivative in Fourier space writes as:

$$
\hat{D}_{\theta}^{2}= \begin{cases}-\operatorname{diag}\left(\mathrm{k}^{2}\right) & \text { if } \quad k=1, \ldots, \frac{K}{2}, \\ -\operatorname{diag}\left(\left(\mathrm{k}-\left(\frac{\mathrm{K}}{2}+1\right)\right)^{2}\right) & \text { if } \quad k=\frac{K}{2}+1, \ldots, K .\end{cases}
$$


Fast Fourier Transform algorithm (FFT) based on Eq. (9) is not yet available. Consequently, the Fourier transform in the azimuthal direction is performed here by matrix products using the subroutine DGEMM of the Blas (Basic Linear Algebra Subprograms) library. Additional cost is expected since for the matrix multiplication transform the cost behaves as $N^{2}$ with respect to $O(N \log N)$ for FFT. However, as mentioned in Boyd [4], the actual costs must be significantly different since it is known that they are strongly grid and hardware dependents (see on Figure 10.2 in Boyd [4]). Several tests have been performed for a grid varying from $K=8$ to $K=192$. The ratio of the cost of the Fourier transform with respect to JMFFT available on the NEC SX8 at IDRIS-CNRS computing center varies from 0.8 to 7.1 that involves a ratio varying from 0.83 to 1.70 for one global time iteration. When vector lengths are not equal to $2^{p} 3^{q} 5^{q}$, the performances become comparable as expected by Temperton algorithms [21]. It should be noted however that the angular shift in the azimuthal discretization leads to a double resolution in physical space.

\subsection{Projection scheme for time-discretization and space approximation}

The velocity-pressure coupling is solved using the 3D projection algorithm proposed by Raspo et al. [18]. It is based on a semi-implicit second-order scheme that combines an implicit backward Euler scheme for the diffusive terms and an explicit Adams-Bashforth extrapolation for the convective nonlinear terms as expressed below:

$$
\begin{gathered}
\frac{3 \mathbf{V}^{n+1}-4 \mathbf{V}^{n}+\mathbf{V}^{n-1}}{2 \partial t}+2\left(\mathbf{V}^{\mathbf{n}} \cdot \nabla\right) \mathbf{V}^{n}-\left(\mathbf{V}^{n-1} \cdot \nabla\right) \mathbf{V}^{n-1}= \\
-\nabla P^{n+1}+\frac{1}{R e} \Delta \mathbf{V}^{n+1}+\mathbf{F}^{n+1} \text { in } \mathrm{D} \\
\nabla \cdot \mathbf{V}^{n+1}=0 \text { in } \mathrm{D} \\
\mathbf{V}^{n+1}=\mathbf{W}^{n+1} \text { on } \partial \mathrm{D}
\end{gathered}
$$

Like in classical projection scheme, a velocity predictor is calculated from Eq. (13), but here the pressure term $P^{n+1}$ is replaced by a pressure predictor, $\phi$ formerly calculated at each time step. This pressure predictor is computed by means of the elliptic equation obtained from Eq. (13) by using Eq. (14), 
associated to Neumann boundary conditions obtained by projecting Eq. (13) on the normal vector to $\partial D$. Projection schemes induce a spurious slip velocity on the boundary since the tangential boundary conditions on the velocity are not prescribed in the projection step. This slip velocity is expressed in $3 \mathrm{D}$ as $V_{s}=n \times\left(\left(V^{n+1}-W^{n+1}\right) \times n\right)$ where $n$ is the unit normal vector to $\partial D$. By allowing correct temporal evolution of the normal pressure gradient at the boundaries during the time integration, numerical results [18] have shown that such calculations significantly improved the accuracy of the scheme and particularly reduces this slip velocity of one order to $O\left(\delta t^{3}\right)$.

All variables $\Psi$ are spatially discretized using a collocation-Chebyshev method for both non-homogeneous directions $(r, z)$ and a Fourier-Garlekin approximation in the azimuthal direction $\theta$. Numerical solution is thus sought under the form of the following truncated series:

$$
\Psi_{N K M}(\bar{r}, \theta, \bar{z})=\sum_{k=1}^{K} \sum_{n=0}^{N} \sum_{m=0}^{M} \hat{\Psi}_{n k m} T_{n}(\bar{r}) T_{m}(\bar{z}) \Phi_{k}(\theta)
$$

where $T_{n}$ and $T_{m}$ are Chebyshev polynomials of highest degree $N$ and $M$ respectively. $(\bar{r}, \bar{z})$ denote the dimensionless space variables $(r, z)$ mapped into the square $[-1,1] \times[-1,1]$, a requisite for the use of Chebyshev polynomials. $N$ and $M$ correspond to the number of Gauss-Lobatto collocation points defined as the extrema of the Chebyshev polynomials of highest degrees $N$ and $M . \Phi_{k}(\theta)$ are the trigonometric basis functions defined in Eq. (9) and $K$ defines the cut-off frequency of the Fourier series that corresponds to $K / 2$ modes. The explicit non-linear terms are calculated using the pseudospectral techniques described in [21] where the derivatives in each space direction are calculated in the spectral space and the products are performed in the physical one. The derivatives of implicit diffusion terms are all performed in physical space using matrix products.

The coupling between the first two components of the vectorial cylindrical Laplacian (Eqs. $(4,5)$ ) usually requires a splitting of the operator in an implicit and an explicit part that leads to time-step limitations. Therefore, the following variable transformation [22] is introduced:

$$
u_{+}=u+i v, \quad u_{-}=u-i v \quad\left(i^{2}=-1\right)
$$


that makes diagonal the Laplacian:

$$
\begin{array}{r}
(\Delta V)_{+}=\left(\nabla^{2}-\frac{1}{r^{2}}+\frac{2 i}{r^{2}} \frac{\partial}{\partial \theta}\right) u_{+} \\
(\Delta V)_{-}=\left(\nabla^{2}-\frac{1}{r^{2}}-\frac{2 i}{r^{2}} \frac{\partial}{\partial \theta}\right) u_{-} \\
(\Delta V)_{z}=\nabla^{2} w
\end{array}
$$

Such a transformation requires the reorganization in the complex plane of the Fourier coefficients matrix $\widehat{\Psi}$ as follows:

$$
\widehat{\Psi}= \begin{cases}\left(\widehat{\Psi}_{j, \frac{K}{2}+k}\right) & , k=1, \\ \left(\widehat{\Psi}_{j, \frac{K}{2}+k}+i \widehat{\Psi}_{j, k-1}\right) & , k=2, \ldots, K / 2\end{cases}
$$

for $1 \leq j \leq N \times M$. It must be noted that the Fourier mode $K / 2$ has been set to zero [21] as previously mentioned. According to Eq. (17), the spectral matrices $\widehat{u}_{+}$and $\widehat{u}_{-}$write:

$$
\widehat{u}_{+}=\widehat{u}+i \widehat{v}, \quad \widehat{u}_{-}=\widehat{u}-i \widehat{v},
$$

that is to say:

$$
\begin{aligned}
& \widehat{u}_{+}=\left\{\begin{array}{l}
\left(\widehat{u}_{j, \frac{K}{2}+k}+i \widehat{v}_{j, \frac{K}{2}+k}\right), k=1, \\
\left(\widehat{u}_{j, \frac{K}{2}+k}-\widehat{v}_{j, k-1}\right)+i\left(\widehat{u}_{j, k-1}+\widehat{v}_{j, \frac{K}{2}+k}\right), k=2, \ldots, K / 2
\end{array}\right. \\
& \widehat{u}_{-}=\left\{\begin{array}{l}
\left(\widehat{u}_{j, \frac{K}{2}+k}+i \widehat{v}_{j, \frac{K}{2}+k}\right), k=1, \\
\left(\widehat{u}_{j, \frac{K}{2}+k}+\widehat{v}_{j, k-1}\right)+i\left(\widehat{u}_{j, k-1}-\widehat{v}_{j, \frac{K}{2}+k}\right), k=2, \ldots, K / 2
\end{array}\right.
\end{aligned}
$$

Finally, the coefficients $\widehat{u}_{j, \frac{K}{2}+k}, \widehat{u}_{j, k-1}, \widehat{v}_{j, \frac{K}{2}+k}$ and $\widehat{v}_{j, k-1}$ write for $1 \leq k \leq$ $K / 2$ : 


$$
\begin{cases}\widehat{u}_{j, \frac{K}{2}+k}=\operatorname{Re}\left(\frac{\widehat{u}_{+}+\widehat{u}_{-}}{2}\right), & \widehat{u}_{j, k-1}=\operatorname{Im}\left(\frac{\widehat{u}_{+}+\widehat{u}_{-}}{2}\right) \\ \widehat{v}_{j, \frac{K}{2}+k}=\operatorname{Im}\left(\frac{\widehat{u}_{+}-\widehat{u}_{-}}{2}\right), & \widehat{v}_{j, k-1}=\operatorname{Re}\left(\frac{\widehat{u}_{-}-\widehat{u}_{+}}{2}\right)\end{cases}
$$

The time-discretization and the Fourier-Galerkin approximation described above lead to a set of elliptic equations for each Fourier wave to be solved in a 2D domain depending on the two non-periodic directions $(r, z)$. Successive 2D Helmholtz and Poisson equations can be written as:

$$
\Delta_{r z}^{(\Psi)} \widehat{\Psi}_{k}-\sigma^{(\Psi)} \widehat{\Psi}_{k}=\widehat{S}_{k}
$$

for $1 \leq k \leq K / 2 . \Delta_{r z}^{(\Psi)}$ and $\sigma^{(\Psi)}$ are given for the different variables by:

$$
\begin{gathered}
\Delta_{r z}^{(\Psi)}=\nabla^{2}-\frac{1}{r^{2}}+\frac{2 i}{r^{2}} \frac{\partial}{\partial \theta} \quad \text { for } \quad \Psi=\mathrm{u}_{+}, \\
\Delta_{r z}^{(\Psi)}=\nabla^{2}-\frac{1}{r^{2}}-\frac{2 i}{r^{2}} \frac{\partial}{\partial \theta} \quad \text { for } \quad \Psi=\mathrm{u}_{-}, \\
\Delta_{r z}^{(\Psi)}=\nabla^{2} \quad \text { for } \quad \Psi=(\mathrm{w}, \mathrm{P}, \phi), \\
\sigma^{(\Psi)}(k, r)=\frac{(k+1)^{2}}{r^{2}}+\frac{3 R e}{2 \delta t} \quad \text { for } \quad \Psi=\mathrm{u}_{+}, \\
\sigma^{(\Psi)}(k, r)=\frac{(k-1)^{2}}{r^{2}}+\frac{3 R e}{2 \delta t} \quad \text { for } \quad \Psi=\mathrm{u}_{-}, \\
\sigma^{(\Psi)}(k, r)=\frac{k^{2}}{r^{2}}+\frac{3 R e}{2 \delta t} \quad \text { for } \quad \Psi=\mathrm{w}, \\
\sigma^{(\Psi)}(k, r)=\frac{k^{2}}{r^{2}} \quad \text { for } \quad \Psi=(\mathrm{P}, \phi)
\end{gathered}
$$

The spectral derivatives of $\widehat{\Psi}_{k}$ in radial and axial directions are expressed in terms of the values of $\widehat{\Psi}_{k}\left(r_{i}, z_{j}\right)$ at other collocation points with appropriate coefficients $d \bar{r}_{i j}^{(p)}$ and $d \bar{z}_{j i}^{(p)}[21]$ as follows:

$$
\frac{\partial^{p} \widehat{\Psi}_{k}}{\partial r^{p}}\left(r_{i}, z_{j}\right)=\sum_{i=0}^{N}\left(\frac{1}{G}\right)^{p} d \bar{r}_{i j}^{(p)} \widehat{\Psi}_{k}\left(r_{i}, z_{j}\right)
$$




$$
\frac{\partial^{p} \widehat{\Psi}_{k}}{\partial z^{p}}\left(r_{i}, z_{j}\right)=\sum_{j=0}^{M} d \bar{z}_{j i}^{(p)} \widehat{\Psi}_{k}\left(r_{i}, z_{j}\right) \quad \text { with } \quad \mathrm{p}=1,2
$$

that can be expressed in matrix notation as:

$$
\begin{array}{r}
\frac{\partial^{p} \widehat{\Psi}_{k}}{\partial r^{p}}\left(r_{i}, z_{j}\right)=D_{r}^{(p)} \Psi \\
\frac{\partial^{p} \widehat{\Psi}_{k}}{\partial z^{p}}\left(r_{i}, z_{j}\right)=\Psi\left(D_{z}^{(p)}\right)^{T}
\end{array}
$$

If we denote $S$ the matrix $\left[\widehat{S}_{k}\left(r_{i}, z_{j}\right)\right]\left(z_{j}=\cos (i \pi / M), 0 \leq j \leq M\right)$, Eq. (26) can be written in the matrix form:

$$
A \Psi+\Psi B^{T}=S
$$

with $A=D_{r}^{(2)}-\frac{1}{r} D_{r}^{(1)}-\sigma^{(\Psi)} I$ and $B=\left(D_{z}^{(2)}\right)$. It is noted that only $B$ does not depend on the harmonic $k$. The Dirichlet boundary conditions are taken into account in $S$ while the Neumann boundary conditions appear in $A$ or $B$ according to the direction. These $2 \mathrm{D}$ algebraic linear systems are solved directly using a full diagonalization technique for each Fourier mode [18].

It should be noted that the matrix $A$ may contain several complex eigenvalues on the three-dimensional Fourier modes that are easily taken into account by the formalism introduced in Eq. (21). This number of complex eigenvalues is mesh dependent and increases with finer meshes in radial and azimuthal directions.

\section{Method accuracy and validation}

The method is validated for flows in shrouded cylindrical rotor-stator cavities of aspect ratio $G=2 R / H$. The fluid of kinematic viscosity $\nu$ is driven by the rotation of the top disc at constant speed $\Omega$, the other walls being at rest. The characteristic scales for space, time and velocity are $H$, $\Omega^{-1}$ and $\Omega R$ respectively. The main flow is governed also by the rotational Reynolds number defined as: $R e=\Omega R^{2} / \nu$. 


\subsection{Method accuracy}

The accuracy of the method is checked by using the following steady $(\beta=0)$ and unsteady $(\beta=1)$ divergence-free velocity field $\mathbf{V}_{\mathbf{e}}=\left(u_{e}, v_{e}, w_{e}\right)$ and pressure $P_{e}$ for $(\bar{r}, \theta, \bar{z}) \in[-1,1] \times[0,2 \pi[\times[-1,1]$ :

$$
\begin{array}{r}
u_{e}(\bar{r}, \theta, \bar{z}, t)=\frac{1}{2 \pi}(\sin (\pi \overline{\mathrm{r}}))^{2} \sin (2 \pi \overline{\mathrm{z}}) \cos (\theta)\left(\beta \cos (8 \pi \mathrm{t})^{2}+1\right) \\
v_{e}(\bar{r}, \theta, \bar{z}, t)=-\frac{1}{2 \pi}(\sin (\pi \overline{\mathrm{r}}))^{2} \sin (2 \pi \overline{\mathrm{z}}) \sin (\theta)\left(\beta \cos (8 \pi \mathrm{t})^{2}+1\right) \\
w_{e}(\bar{r}, \theta, \bar{z}, t)=-\frac{1}{2 \pi G}(\sin (2 \pi \overline{\mathrm{r}})) \sin (\pi \overline{\mathrm{z}})^{2} \cos (\theta)\left(\beta \cos (8 \pi \mathrm{t})^{2}+1\right) \\
P_{e}(\bar{r}, \theta, \bar{z}, t)=[\cos (\pi \overline{\mathrm{r}})+\cos (\pi \overline{\mathrm{z}})] \cos (\theta)\left(\beta \cos (8 \pi \mathrm{t})^{2}+1\right)
\end{array}
$$

The results have been obtained for a cavity of aspect ratio $G=10$ and at a Reynolds number $R e=250$. The velocity field $\mathbf{V}_{\mathbf{e}}$ satisfies homogeneous Dirichlet boundary conditions. The space and time accuracy is evaluated by computing the discrete error at the inner collocation points for $\Psi_{e}=$ $\left(u_{e}, v_{e}, w_{e}, P_{e}\right)$ defined on an ansatz of $L_{2}$-norm:

$$
E r=\left(\frac{1}{N_{\text {tot }}} \sum_{k=1}^{K} \sum_{i=1}^{N-1} \sum_{j=1}^{M-1}\left[\Psi_{e}\left(r_{i}, \theta_{k}, z_{j}, t\right)-\Psi_{N K M}\left(r_{i}, \theta_{k}, z_{j}, t\right)\right]^{2}\right)^{\frac{1}{2}}
$$

where $N_{\text {tot }}=(N-1) \times(M-1) \times K$.

\subsubsection{Exact steady solution}

The computations are initialized using the disturbed velocity field:

$$
\mathbf{V}_{0}=\mathbf{V}_{e}+\frac{1}{200 \pi^{2}} * \mathbf{V}_{e}
$$

The time step is fixed at $\delta t=5 \times 10^{-3}$. The steady solution is assumed to be obtained when the residuals $\operatorname{Res}(\Psi)=\max _{i, k, j} \mid \Psi_{N K M}^{n}\left(r_{i}, \theta_{k}, z_{j}\right)-$ $\Psi_{N K M}^{n-1}\left(r_{i}, \theta_{k}, z_{j}\right) \mid / \delta t$ are below $10^{-10}$. The spectral convergence of the method is obtained for all variables. The error $E r$ is reported for the velocity component $u$ and for the pressure $P$ in Figure 2(a). The error decays exponentially to the zero machine for $N=M=K=28$ then slightly increases due to the round-off error as usually observed [18]. 


\subsubsection{Exact time-dependent solution}

For $\beta=1, \Psi_{e}$ is time periodic with a period $T=0.125$. The computations are initialized with $V_{0}=V_{e}(t=0)$. The mesh is fixed at $N=M=K=40$ and the time step is decreased from $\delta t=5 \times 10^{-3}$ to $5 \times 10^{-5}$. The Neumann boundary conditions are used for the pressure. When the periodic state is reached, the time accuracy is evaluated by computing the maximum of $\operatorname{Er}(t)$ for $\Psi=(u, v, w, P)$ over the computational time. The results show for all variables that the method is second-order accurate. The maximum of the error $\operatorname{Er}(t)$ is reported for the radial velocity component $u$ and for the pressure $P$ in Figure 2(b).

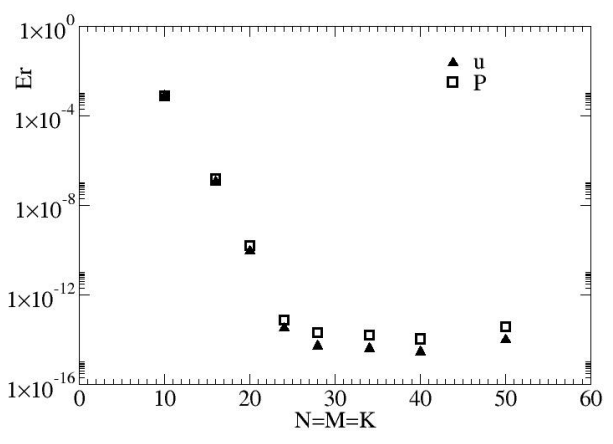

(a)

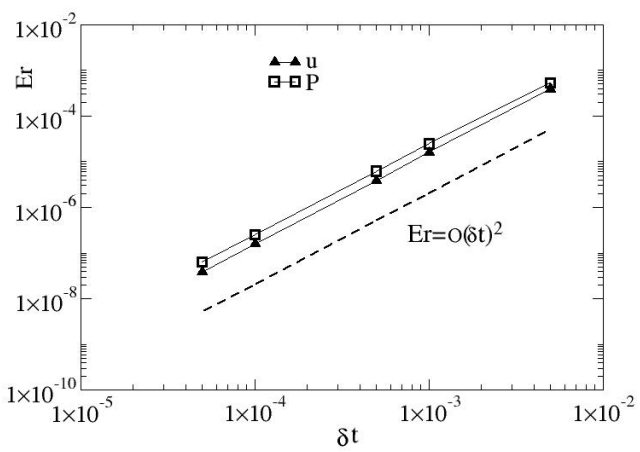

(b)

Figure 2: Space and time accuracy of the method for exact solutions. Evolution of the error at the inner collocation points $E r$ for the radial velocity component $u$ and for the pressure $P$ : a) versus the polynomial degrees $N=M=K, \mathrm{~b}$ ) versus the time step $\delta t$.

\subsection{Validation with reliable results of the literature}

Solutions are now obtained in tall and thin inter-disc rotor-stator cavities. Such flows produce physical phenomena of weak intensity with respect to the base flow both in the vicinity of the axis and in the boundary layers at large radii that still today challenge all numerical solvers. Velocity boundary conditions are no-slip on all walls and $v=\bar{r}$ at the rotating disc. The singularity at the junction between the stationary cylinder and the rotor is appropriately regularized by using an exponential smoothing of the $v$-profile as proposed in [14], $v_{\mu}=\exp (-(\mathrm{z}-1) / \mu)$ with $\mu=0.006$.

In such cavities the laminar base flow is axisymmetric and three dimensional. Centrifugal effects produce a secondary vortical flow in the meridian 
plane $(r, z)$. The rotating disc plays the role of a centrifugal fan by which fluid is sucked towards the disc in the vertical direction and thrown radially outwards near the disc (usually referred as Ekman pumping [23]). Due to continuity and to centrifugal pressure created by the rotation of the fluid, a radial inflow appears near the stationary disc as illustrated in Figure 3.

In the inter-disc cavity, the two boundary-layers along the discs are separated by a large non-viscous geostrophic core in which the fluid has a nearly solid-body rotation and zero flow in the meridian plane as illustrated in Figure 3(b). This is a consequence of the Taylor-Proudman theorem. This theorem is obtained by taking the curl of conservation equations that reduce to a balance between Coriolis force and pressure gradient in a rotating frame of reference attached to the lid when Coriolis force dominates inertial and viscous forces.

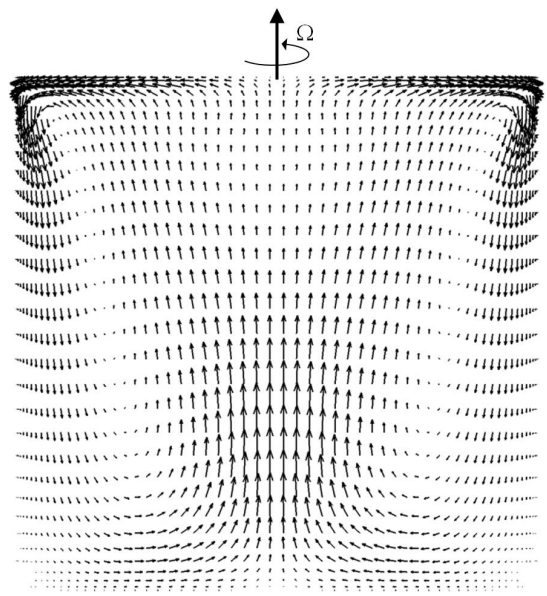

(a) $R e=10^{3}, G=1$

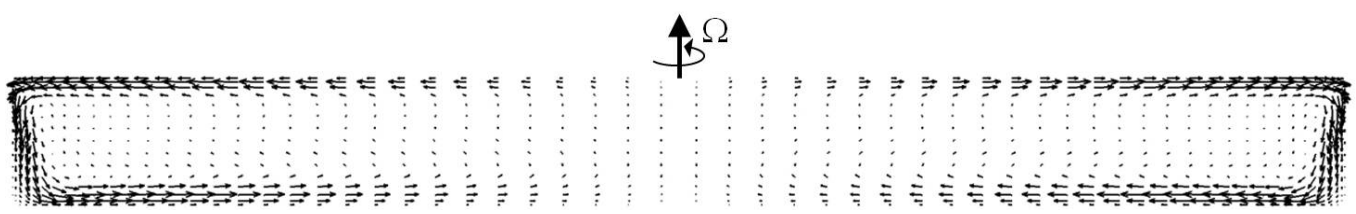

(b) $R e=10^{4}, G=10.26$

Figure 3: Base flows in a tall and a thin rotor-stator cavities showing the secondary crossflow in the meridian $(r, z)$-plane. Projected velocity vector fields. 


\subsubsection{Vortex breakdown in tall rotor-stator cylinders}

The confined vortex flow of Figure 3(a) can undergo breakdown for certain combinations of $(G, R e)$. The vortex breakdown phenomenon occurs as an abrupt change in the structure of the vortex core around the cylinder axis and typically develops downstream into a stagnation zone in the flow. Escudier [24] showed experimentally that several recirculation bubbles may exist on the main vortex axis and mapped out the steady and unsteady flow regions in the $(G, R e)$-plane.

The accuracy of the algorithm to simulate secondary vortices around $r=$ 0 is shown on simulations covering both axisymmetric (steady and unsteady) and three-dimensional vortex breakdown. In all axisymmetric cases the mesh size is fixed at $126 \times 65$ collocation points in the radial and axial directions respectively and the time step is equal to $10^{-2}$. The three-dimensional regime is investigated using 24 Fourier modes.

In stationary axisymmetric flows, the vortex core undergoes breakdown for $(G=1, R e>1500)$ in agreement with Escudier's observations [24]. The number and the position of the recirculation bubbles are accurately predicted. For $(G=1, R e=1850)$ the two axisymmetric steady bubbles (Figure 4$)$ are well characterized as shown by the agreement with results of the literature in Table 1.

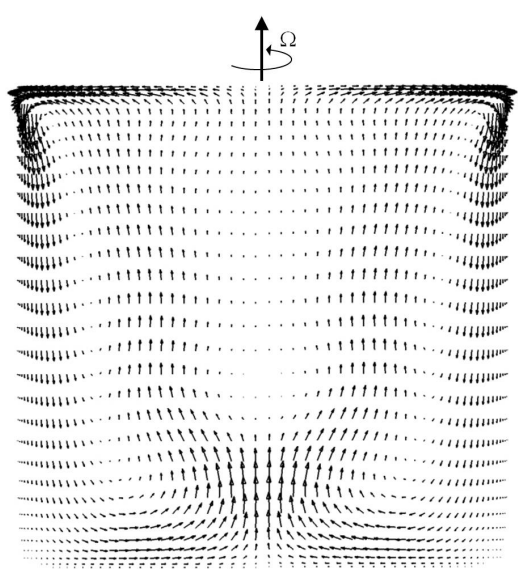

(a)

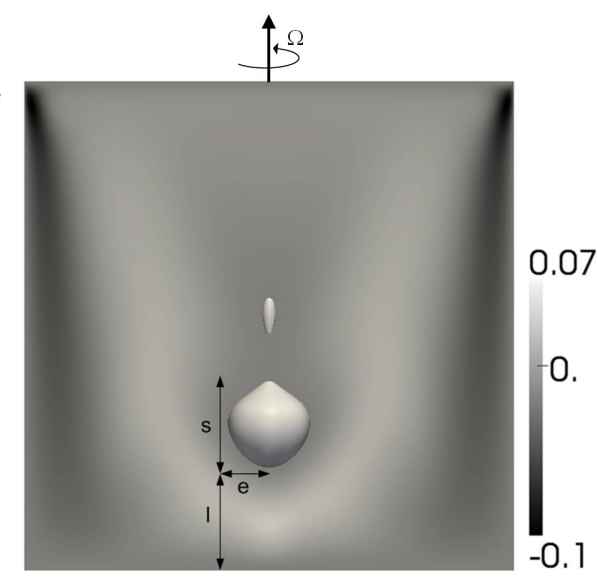

(b)

Figure 4: Steady axisymmetric vortex breakdown at $(G=1, R e=1850)$ composed by two bubbles. (a) Projected velocity vector field in a $(r, z)$ plane and (b) isolines of the axial velocity component $w$. The recirculation flow region is characterized by the $w=0$ isosurface. $s, l, e$ are three characteristic lengths of the vortex breakdown. 


\begin{tabular}{ccccc}
\hline \hline & $R e$ & $\mathrm{l} / \mathrm{H}$ & $\mathrm{s} / \mathrm{H}$ & $\mathrm{e} / \mathrm{H}$ \\
\hline Experiments of Escudier [24] & 1854 & & & \\
bubble 1 & & 0.21 & 0.16 & 0.113 \\
bubble 2 & & 0.52 & 0.07 & 0.019 \\
\hline $\begin{array}{c}\text { DNS of Sotiropoulos and Ventikos [25] } \\
\text { bubble 1 }\end{array}$ & & 0.21 & 0.176 & 0.113 \\
bubble 2 & & 0.464 & 0.081 & 0.02 \\
\hline DNS of Serre et al [26] & 1850 & & & \\
bubble 1 & & 0.208 & 0.179 & 0.120 \\
bubble 2 & & 0.482 & 0.080 & 0.025 \\
\hline present simulation & 1854 & & & \\
bubble 1 & & 0.2106 & 0.1763 & 0.0841 \\
bubble 2 & & 0.4812 & 0.0756 & 0.0151 \\
\hline \hline
\end{tabular}

Table 1: Comparison of the characteristic lengths of the stationary vortex breakdown in a cylinder of aspect ratio $G=1$ at $R e=1850$. Bubble 1 and bubble 2 correspond to the lower and the upper recirculation regions, respectively. The characteristic lengths $l, s$ and $e$ are defined in Figure 4(b).

In unsteady axisymmetric regimes, the solutions are accurate as well. The unsteadiness corresponds to an oscillation of the two bubbles along the axis as shown on Figure 5 for $(G=0.8, R e=2750)$. The dimensionless time period is $36.2 \Omega^{-1}$ that matches the value measured by Stevens et al. [27], $36 \Omega^{-1}$.

Unsteady three-dimensional regimes show a chaotic temporal behavior associated with an asymmetric vortex breakdown precessing around the axis in the same direction as the main flow, as illustrated in Figure 6 for $(G=$ $0.5, R e=6500)$. The solution corresponds to the 'S-shaped' regime first noted by Escudier [24] and it is similar to the former one described in Ref. [26].

\subsubsection{Inter-disc rotor-stator flows at transitional Reynolds numbers}

When the cavity height $H$ is assumed to be large with respect to the characteristic viscous length $\delta=(\nu / \Omega)^{0.5}$, the base flow (Figure 3(b)) produces adjacent coupled flow regions that are radically different in terms of the flow properties and the thickness scales, involving very challenging simulations [19]. The rotor boundary layer is very close to the von Kármán solution for a single rotating disc boundary layer; it is known to be stable and very robust to finite disturbances [28]. The stator boundary layer has a profile that matches with the Bödewadt solution for a single stationary disc topped 


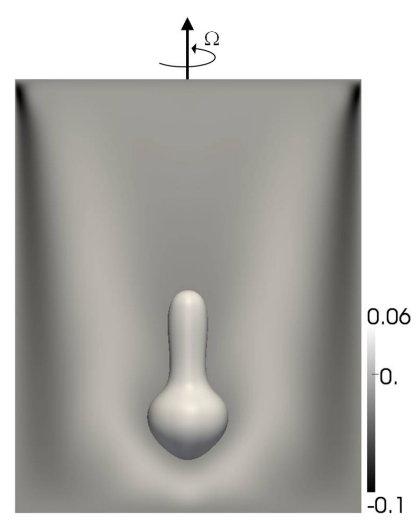

(a) $\mathrm{t}=2512 \Omega^{-1}$

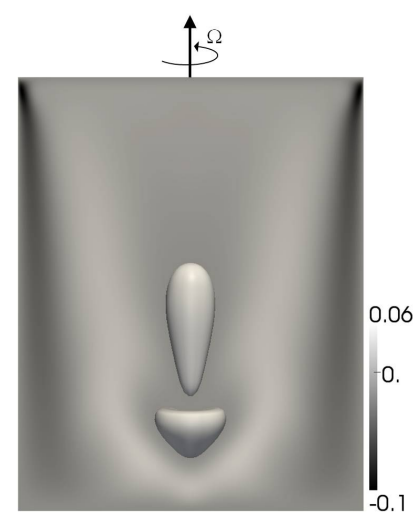

(b) $\mathrm{t}=2524 \Omega^{-1}$

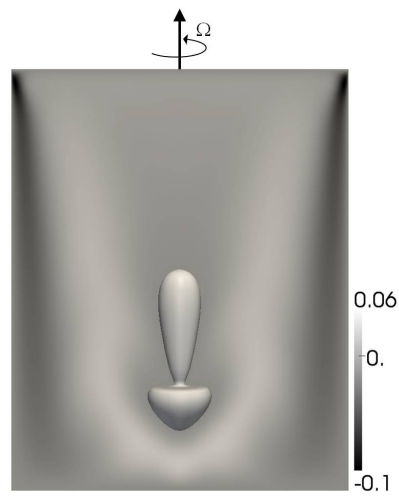

(c) $\mathrm{t}=2536 \Omega^{-1}$

Figure 5: Oscillating axisymmetric vortex breakdown of period $36.2 \Omega^{-1}$. Snapshots of $w=0$ iso-surfaces and iso-contours of $w$ in a $(r, z)$ plane for $(G=0.8, R e=2750)$.

by an ambient flow in solid-body rotation. This boundary layer is very unstable to crossflow instability characterized by multiarmed spiral waves. It is also known to be sensitive to finite disturbances with axisymmetric circular waves travelling radially inward [29]. The aspect ratio $G$ is chosen equal to 17.5 as in the documented experiments of Schouveiler et al. [30]. The grid size is fixed at $(N, K, M)=126 \times 128 \times 33$ points in the radial, azimuthal and axial directions respectively and the time step is fixed equal to $2 \times 10^{-3}$.

The response of the flow to spin up is analyzed for Reynolds numbers in a range $[3000,20900]$ where only the Bödewadt layer is expected to be unstable. Typical numerical results are summarized on Figure 7 and show a very good agreement with the literature. When increasing rotation rate from a steady state, the code is able to predict transient circular rolls within the stationary disc boundary layer. In agreement with the literature (see for example [31]) they propagate radially inward and are amplified to about mid-radius before decaying as they approach the axis (Figure 7(b)). Similar structures have been also observed experimentally in the same cavity by Schouveiler et al. [30] for $R e \geq 10500$ and for an other aspect ratio [32, 33].

The response of the flow to an impulsive 3D perturbation can give rise to a three-dimensional self-sustained saturated wave characterized by spiral arms at the periphery of the disc that extend to about mid-radius, as illustrated here on Figure 7 at $R e=20900$. In such situation, they coexist with circular rolls during a transient state but only spiral arms remain, the 


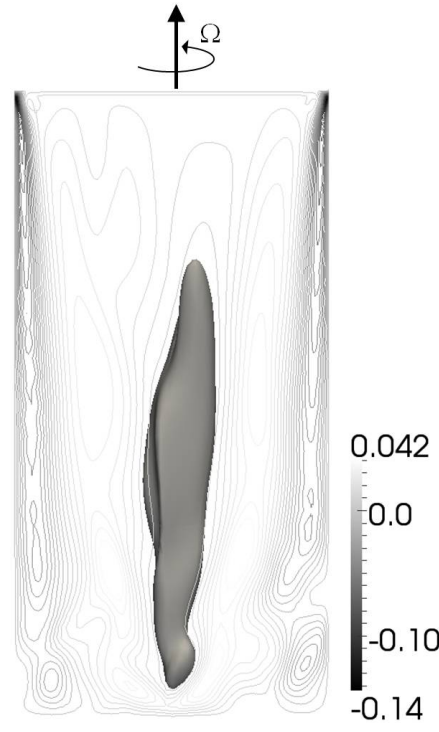

(a) $\mathrm{t}=2484 \Omega^{-1}$

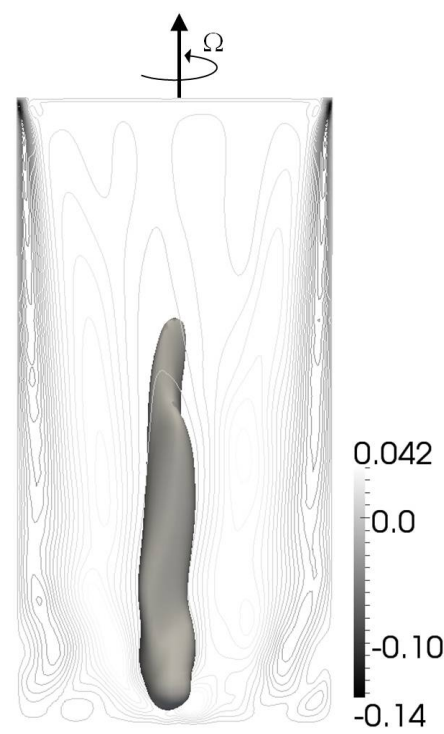

(d) $\mathrm{t}=2544 \Omega^{-1}$

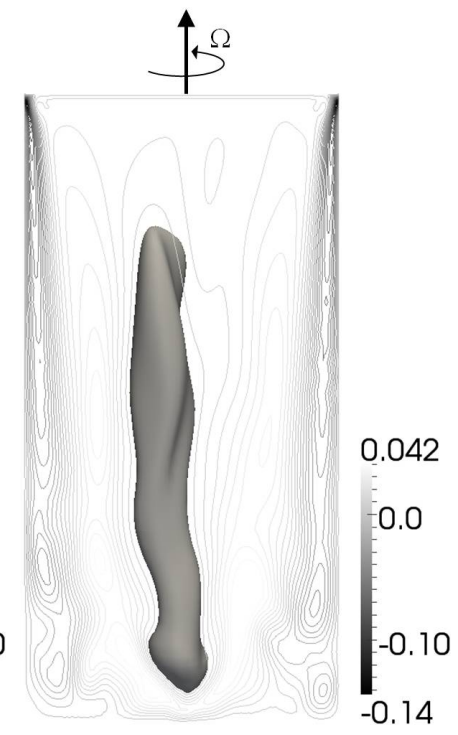

(b) $\mathrm{t}=2504 \Omega^{-1}$

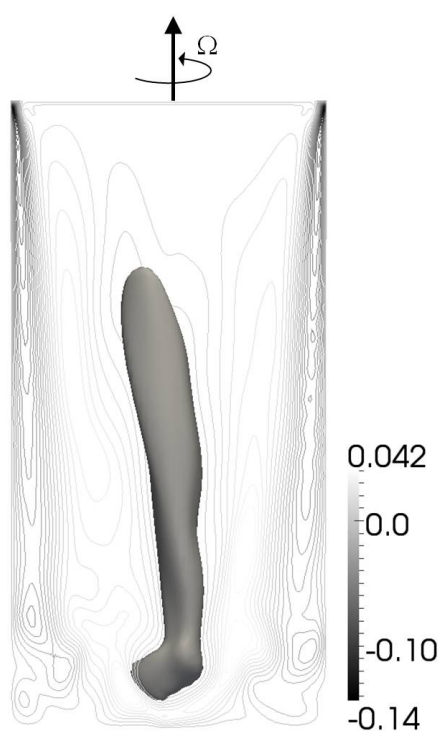

(e) $\mathrm{t}=2564 \Omega^{-1}$

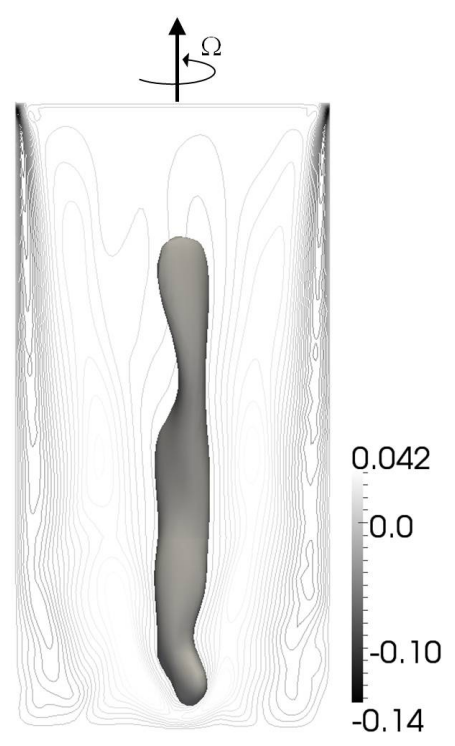

(c) $\mathrm{t}=2524 \Omega^{-1}$

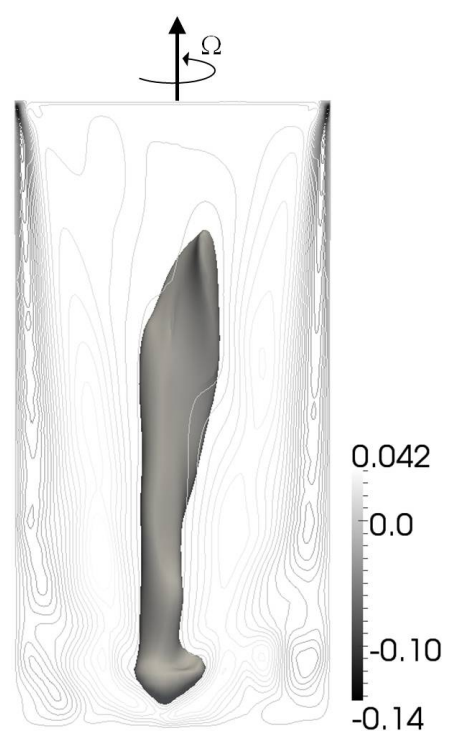

(f) $\mathrm{t}=2584 \Omega^{-1}$

Figure 6: Unsteady and three-dimensional 'S-shaped' vortex breakdown for $(G=0.5, R e=$ $6500)$. Snapshots of $w=0$ iso-surfaces and iso-lines of the axial velocity component $w$. 


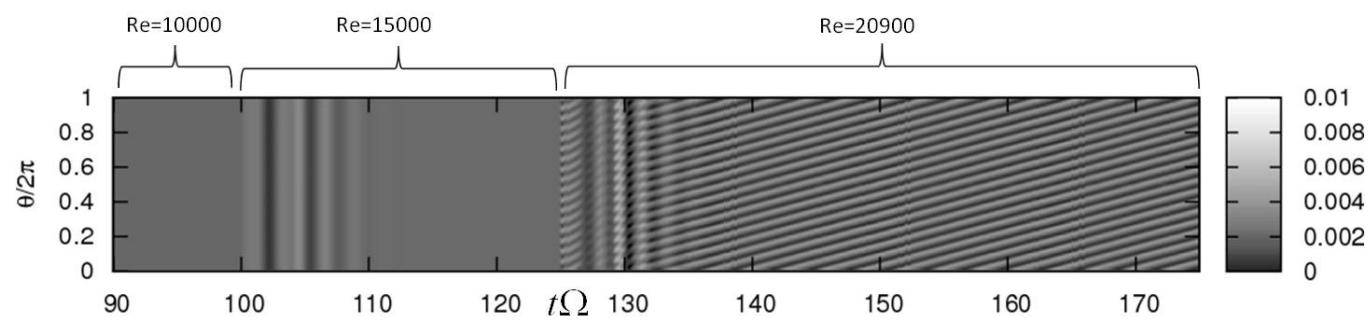

(a)

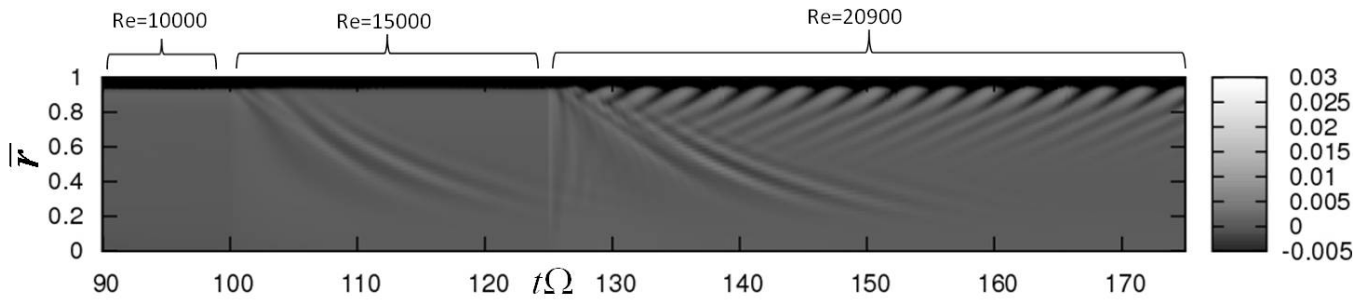

(b)

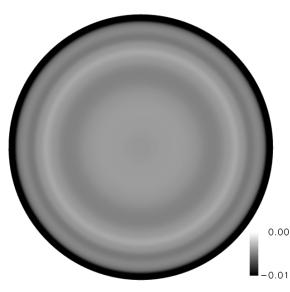

(c) $\mathrm{t}=105 \Omega^{-1}$

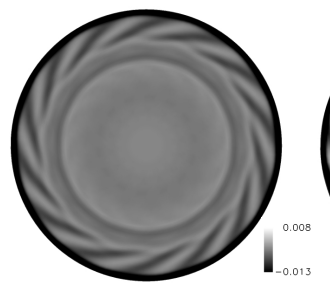

(d) $\mathrm{t}=132 \Omega^{-1}$

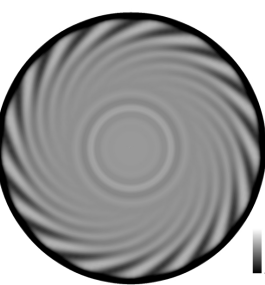

$\mathrm{t}=150 \Omega^{-1}$

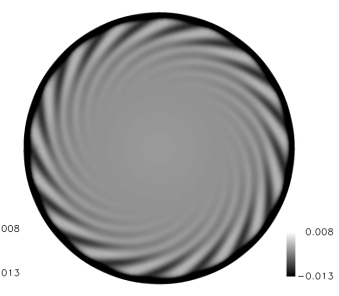

$\mathrm{t}=175 \Omega^{-1}$

Figure 7: Transition to an unstable stator boundary-layer flow at $R e=10000$ and $R e=$ 20900 in a cavity of aspect ratio $G=17.5$. (a,b) Spatio-temporal diagrams showing the dynamics of the instability structures during spin up: (a) $w(\bar{r}=0.809, \bar{z}=-0.773, \theta, t)$ : (b) $w(\bar{r}, \bar{z}=-0.773,0, t)$. (c,d) Instability patterns: (c) transient circular rolls alone at $R e=15000\left(t=105 \Omega^{-1}\right)$ and $(\mathrm{d})$ transient circular rolls and permanent $m=17$ spiral arms at $R e=20900\left(t=132 \Omega^{-1}, t=150 \Omega^{-1}, t=175 \Omega^{-1}\right)$. The disc rotates counter-clockwise. 
circles having disappeared when reaching the vicinity of the axis as shown on Figure 7(b). These rotating spiral arms roll up in the main flow direction with a dimensionless time-period of $3 \Omega^{-1}$ and form a positive angle with the tangential direction equal to $\varepsilon=28.9^{\circ}$ at $\bar{r}=0.809$ (Figure $7(\mathrm{a})$ ).

Such results are in agreement with former DNS results in annular cavity [29] and with experimental observations [30]. Schouveiler et al. [30] show $m=18$ spiral rolls of angle about $\varepsilon=25^{\circ}$ for $R e \geq 20000$. As here, spiral arms are located at the periphery but coexist permanently with circular rolls due to inherent experimental perturbations that act as wavemakers. The difference in the selected azimuthal wavenumbers can be related to the Eckhaus instability mechanism observed in [30] resulting in the exponential growth of wavenumber modulations and, on the nonlinear stage, change of the initial wavenumber towards the optimal value through defects. Present simulations show that a finite band of wavenumbers between $m=14$ and $m=20$ goes unstable and that the selected wavenumber, $m=17$, corresponds here to the maximum growth rate $\alpha$ equal to 0.0388 (Figure 8). According to Figure 8(b) the growth rate of modes $m=16,17,18$ are very close indicating that any of this wavenumbers could saturate the first depending on the history of the flow.

Due to the permanent stable region in the vicinity of the axis, any feedback from the stator on the basic state can be avoided. Then, the stationary disc boundary layer behaves as an unstable, non-parallel opened flow despite the large-scale meridional circulation. As consequence, these behaviours can be interpreted in the frame of the linear stability results of Serre et al. [28] that predict convectively unstable circular waves and absolute three-dimensional waves.

\subsection{Temporal stability limit}

The temporal stability limit of the algorithm has been investigated as a function of spatial discretization and Reynolds number from the simulations presented in sections 4.2.1 and 4.2.2. Time-step requirements are imposed by the advective terms which are treated explicitly. When varying discretization, time-step limit was found to be proportional to $1 / N^{2}$ (Figure $9($ a)) as expected from theoretical analysis [4]. In other words, the semi-implicit algorithm avoids the much stiffer time-step requirements $\left(O\left(1 / N^{4}\right)\right)$ imposed by second derivatives involved in the viscous terms. When increasing Reynolds number for a fixed grid, the time-step decreases very slowly and almost linearly (Figure 9(b)). The larger gap between the two first points corresponds 


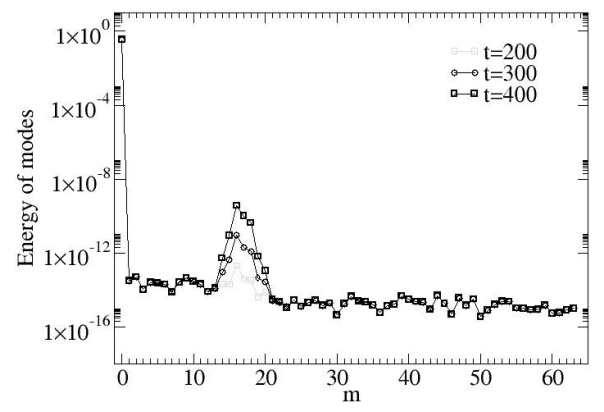

(a)

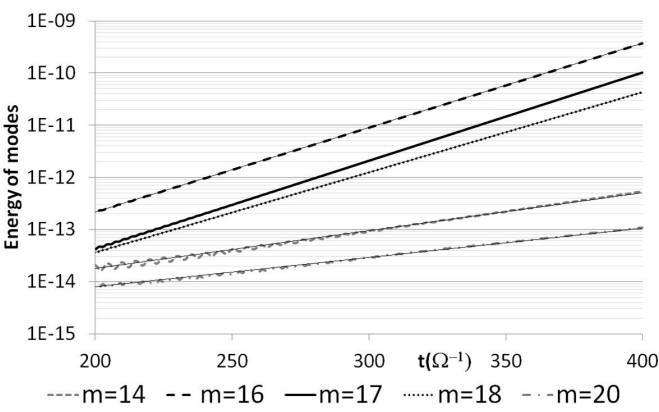

(b)

Figure 8: Flow spectral analysis $(G=17.5, R e=20900)$. (a) Energy spectrum of the solution at three different times. (b) Temporal evolution of the kinetic energy of the unstable modes corresponding to the following growth rates $\alpha$ : $0.0388,0.0373,0.0354,0.0167$ and 0.0129 for $m$ equal to $17,16,18,14$ and 20 , respectively.

to the transition between stable and unstable flow regimes, characterized by the occurrence of convective circular patterns on Figure 7.

\subsection{Performances study}

Performances of the code are provided in Table 2 for simulations running on the Nec SX8 vectorial computer at the CNRS computing center. The memory requirement increases linearly with the mesh. The number of floating-point operations per second (Gflops) slightly increases with the mesh to reach $80 \%$ of the crest performance of the vectorial processor for the largest grid. This shows a good optimization of the code on this processor that is assessed by the low CPU time per degree of freedom of order $O\left(10^{-7}\right)$.

\begin{tabular}{ccccc}
\hline \hline Mesh $(r, \theta, z)$ & Memory $(M b)$ & Time/It $(s)$ & GFlops & Time/DF $(s)$ \\
\hline $126 \times 8 \times 65$ & 144 & $6.26 \mathrm{E}-02$ & 11.37 & $1.91 \mathrm{E}-07$ \\
$126 \times 128 \times 33$ & 592 & $6.86 \mathrm{E}-01$ & 12.2 & $2.58 \mathrm{E}-07$ \\
$196 \times 288 \times 71$ & 3600 & 10.28 & 13.4 & $5.13 \mathrm{E}-07$ \\
\hline \hline
\end{tabular}

Table 2: Code performances on Nec SX8 vectorial computer for three grid sizes. Memory requirement, CPU time by iteration (It), Floating-point operations per second (GFlops), and CPU time by degree of freedom (DF). 


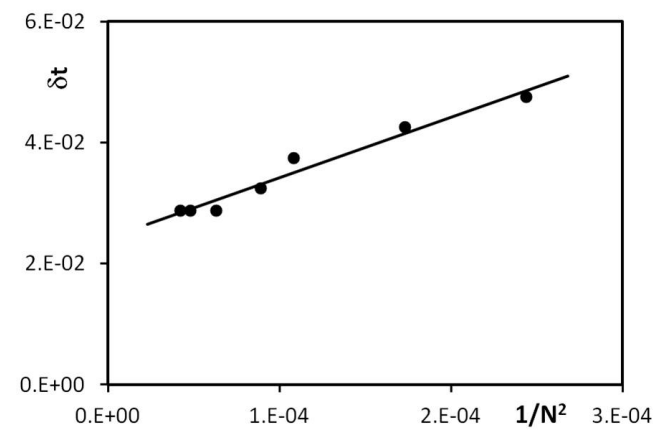

(a)

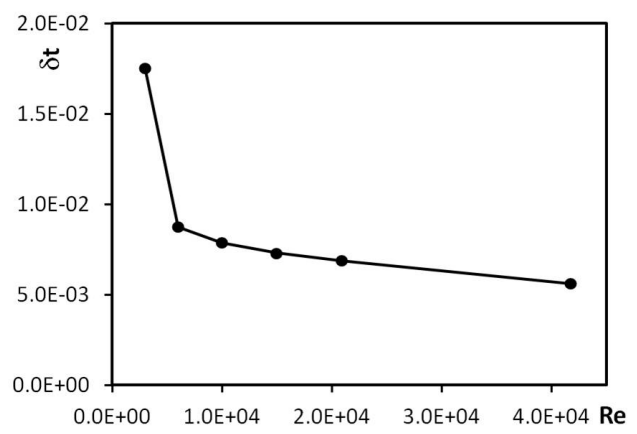

(b)

Figure 9: Temporal stability limit of the algorithm. Variations of the critical time-step $\delta t$ as a function of the number of collocation points $N$ (a) and as a function of Reynolds number (b). The analysis has been carried out from simulations presented in Sections 4.2.1 and 4.2.2 for $G=0.8$ and $R e=2750$ (a) and for $G=17.5$ and $N=126$ (b).

\section{Turbulent rotor-stator cavity flows}

Two LES of turbulent flows are now provided in a tall heated rotating cylinder and in a thin rotor-stator cavity. Such simulations remain a challenge (see a review in Launder et al. [19]), in particular when using spectral approximations that are much less diffusive than low-order ones. For both flows, the method works very well and does not generate any spurious oscillations when small scale energetic eddies are convected near the singularity axis. The present method has been associated to the spectral vanishing viscosity (SVV) technique introduced in cylindrical geometries in Séverac and Serre [34] and successfully applied in annular geometries for Reynolds numbers up to $10^{6}$ in Séverac et al. [35]. This alternative LES formulation is proposed and incorporated into the Navier-Stokes equations for controlling high-wavenumber oscillations by incorporating in the Navier-Stokes equations a well-controlled diffusion. A new diffusion operator $\Delta_{S V V}$ is simply implemented by combining the classical diffusion and the new SVV terms to obtain:

$$
\nu \Delta_{S V V} \equiv \nu \Delta+\nabla \cdot\left(\varepsilon_{N} Q_{N} \nabla\right)=\nu \nabla \cdot S_{N} \nabla
$$

where $\nu$ is the diffusive coefficient and where:

$$
S_{N}=\operatorname{diag}\left\{\mathrm{S}_{\mathrm{N}_{\mathrm{i}}}^{\mathrm{i}}\right\}, \mathrm{S}_{\mathrm{N}_{\mathrm{i}}}^{\mathrm{i}}=1+\frac{\varepsilon_{\mathrm{N}_{\mathrm{i}}}^{\mathrm{i}}}{\nu} \mathrm{Q}_{\mathrm{N}_{\mathrm{i}}}^{\mathrm{i}}
$$


with $\varepsilon_{N_{i}}^{i}$ the maximum of viscosity and $Q_{N_{i}}^{i}$ a $1 \mathrm{D}$ viscosity operator acting in direction $i$, and defined in the spectral space by an exponential function: $\widehat{Q}_{N_{i}}^{i}(\omega)=0$, if $0 \leq \omega \leq \omega_{T}^{i}$ and $\widehat{Q}_{N_{i}}^{i}(\omega)=\exp \left(-\left(\omega-\omega_{T}^{i}\right)^{2} /\left(\omega-\omega_{T}^{i}\right)^{2}\right)$ if $\omega_{T}^{i} \leq \omega \leq \omega_{N}^{i}$, where $\omega_{T}^{i}$ is the threshold after which the viscosity is applied and $\omega_{N}^{i}$ the highest frequency calculated in the direction $i$. Such model is only active for the short length scales and it has been shown that it keeps the spectral convergence [34].

\subsection{Flow in a thin inter-disc rotor-stator cavity at $R e \approx O\left(10^{5}\right)$}

A flow in a cavity of aspect ratio $G=10.26$ is simulated [36]. The grid in the radial, azimuthal and axial directions is $196 \times 288 \times 71\left(\sim 4 \times 10^{6}\right.$ nodes) and it is associated to the time step $\delta t=5 \times 10^{-4}$. Parameterized amplitude and thresholding of the SVV are equal to $\varepsilon_{N}=(1 / N, 1 / N, 1 / N)$ and $\omega_{T}=(\sqrt{N}, \sqrt{N}, \sqrt{N})$ in $(r, \theta, z)$ directions, respectively.

The stator layer is fully turbulent. The accuracy of the algorithm is evaluated on the prediction of the transition to turbulence expected at the periphery of the rotor boundary-layer and for which theoretical results are available [37]. On the stator side, turbulence appears at about $R e=4 \times 10^{4}$ and at $R e=4 \times 10^{5}$ (Figure 10(a)), the highest Reynolds number considered in this study, the flow is fully turbulent characterized by thin structures that tend to be circular and extend to the vicinity of the axis in agreement with experimental observations [38].

On the rotor side, turbulence appears at the periphery of the disc between $R e=2 \times 10^{5}$ and $R e=2.5 \times 10^{5}$. At $R e=4 \times 10^{5}$, numerical results confirm experimental observations of Lingwood [37] showing that the inflexional instability undergoes a transition from convective to absolute instability at a Reynolds number just below the value found for transition to turbulence. A self-sustained saturated wave related to the crossflow instability and with high-wavenumber $m=68$ occurs at large radius $\bar{r} \approx 0.69$, corresponding to Rossby number $R o\left(=\left(\Omega_{F}-\Omega_{d}\right) / \Omega_{d}\right)=-0.72$ and local Reynolds number $R e_{\delta}(=-\bar{r} R o \sqrt{R e})=314$. This well-organized spiral pattern is immediately followed by a very disorganized state defining the transition to turbulence as shown in Figure 10(b). This state is characterized by a sudden increase of the turbulent kinetic energy forming a steep front from a nearly zero level related to numerical noise as shown on Figure 11. Numerical results are able to accurately predict the front location with respect to the predicted convective to absolute instability transition region [37], its slope with respect to the linear 
absolute spatial growth rate of the absolute instability $\left(k_{0, i}=3.6347\right)$, the amplitude and starting point of the saturated wave that agree with the theoretical prediction for a nonlinear global mode of the rotating disc boundary layer. Present results are consistent with the scenario of transition to turbulence obtained in recent theoretical and numerical studies of Pier [39] and Viaud et al. [40]. In this sense, they should interfere in the single-disc debate around the existence of a direct route to turbulence in the rotating disc boundary layer.

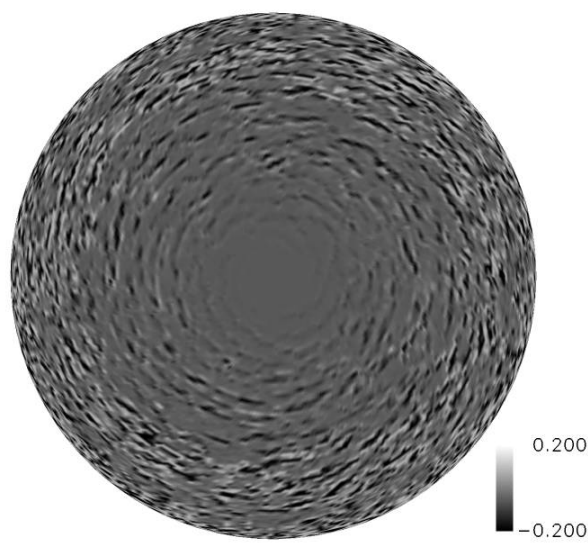

(a) Stator $(\bar{z}=-0.920)$

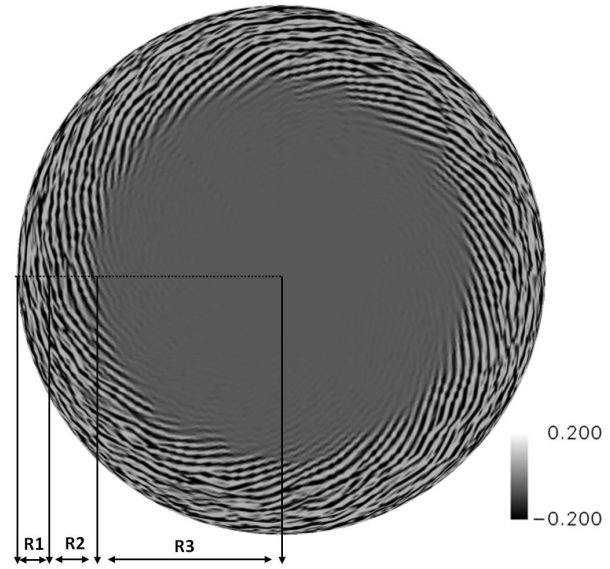

(b) $\operatorname{Rotor}(\bar{z}=0.951)$

Figure 10: Turbulent rotor-stator flow patterns $\left(G=10.26, R e=4 \times 10^{5}\right)$ : (a) Thin quasicircular patterns in the stator layer, (b) stable flow region $\left(R_{1}\right)$ and multiarmed spiral front $(m=68)$ of the absolute mode of crossflow instability $\left(R_{2}\right)$ immediately followed by incipient turbulence $\left(R_{3}\right)$ in the rotor boundary-layer. Iso-lines of the Q-criterion.

5.2. Flow in a tall rotor-stator cylinder heated from below at $R e=10^{5}$ and $R a=10^{6}$

A flow of air (Prandtl number $\operatorname{Pr}(=\nu / \alpha)=0.71)$ is heated from below up to Rayleigh number $R a\left(=\left(\Omega^{2} R H^{3} \Delta T \beta_{0}\right) /(\nu \alpha)\right)=10^{6}$ in the tall cylinder of aspect ratio $G=0.8$. $\alpha$ is the thermal diffusivity, $\beta$ is the thermal expansion coefficient and $\Delta T$ is the temperature difference between the heated bottom disc and the cooled top rotating-disc. The direction of the gravity $g$ is opposite to the unit vector in the axial direction. The temperature $T$ is governed by heat equation resolved as a preliminary step using velocity at time-step 


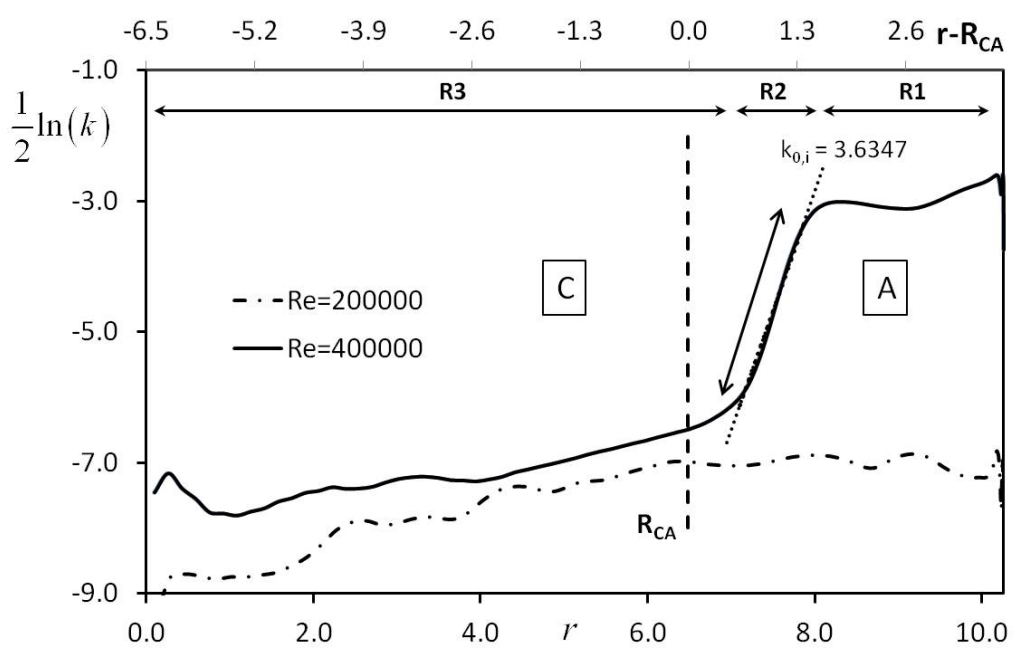

Figure 11: Existence of a steep front located at the convective to absolute instability transition region (C-A). The slope matches the linear absolute spatial growth rate of the absolute crossflow instability, $k_{0, i}=3,6347$. It is immediately followed by a turbulent region. Radial profiles of the turbulence kinetic energy $k$ within the rotor boundary-layer $(\bar{z}=0.951)$ for $G=10.26$ and $R e$ equal to $2 \times 10^{5}$ and $4 \times 10^{5} .\left(R_{1}, R_{2}, R_{3}\right)$ are the regions of space defined on Figure 10 . 
$n-1$. The temperature is made dimensionless using $\Delta T=T_{\max }-T_{\min }$. The bottom and top discs are thermally conducting at $T=+1$ and $T=-1$, respectively, whereas the vertical sidewall is considered as insulating. Mixed convection is modeled through the Boussinesq approximation (Eq. 1). The grid is $126 \times 64 \times 129$ in the radial, azimuthal and axial directions, respectively and time-step is $\delta t=2 \times 10^{-3}$. SVV parameters are identical to Section 5.1.

For these parameters the flow is still dominated by forced convection induced by high rotation, Figure 12. The combined effect of rotation and buoyancy involve small scale energetic vortices that are eventually convected in the vicinity of the axis, as shown in Figures 12(a) and 12(b). Profiles of radial and axial components of the mean velocity (Fig. 12(c)) show that the intensity of the flow along the axis can reach about $10 \%$ of the maximum vertical velocity. Moreover, the flow can cross the cylinder axis, as shown by negative values of the radial component at some places, without involving any spurious numerical oscillations.

\section{Concluding remarks}

A spectrally convergent method is proposed to solve three-dimensional Navier-Stokes equations in cylindrical coordinates for an incompressible fluid. The algorithm avoids pole condition at the center and parity condition in the azimuthal direction. The distribution of the Gauss-Lobatto collocation points avoids an excessive grid clustering about $r=0$, which would be able to lead to an ill-conditioned system with severe time-step restrictions. The algorithm is well-suited for rotating cavity flows where thin unstable boundarylayers develop along the walls. The code is computationally efficient both in terms of CPU time and memory requirement. Numerical solutions match reliable experimental and theoretical results of the literature. Secondary vortices are accurately predicted both when they occur along the cylinder axis in tall cylinders as they appear in thin boundary layers in the inter-disc cavities. As far as the authors know, first LES are provided in rotating cylindrical cavities with no hub. Numerical results show that the method does not generate any spurious oscillations in the vicinity of the cylinder axis and that the algorithm provides quantitative predictions with high-accuracy with respect to theoretical results. 


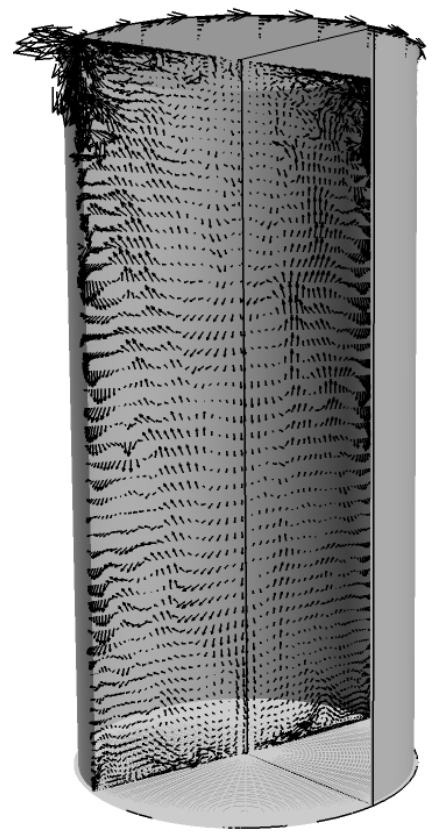

(a)

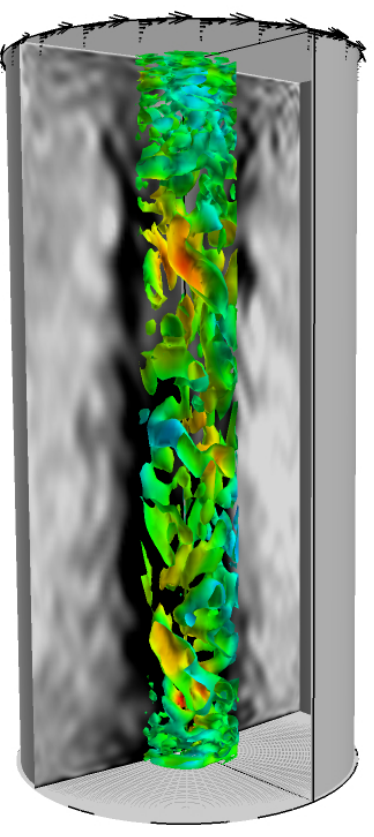

(b)

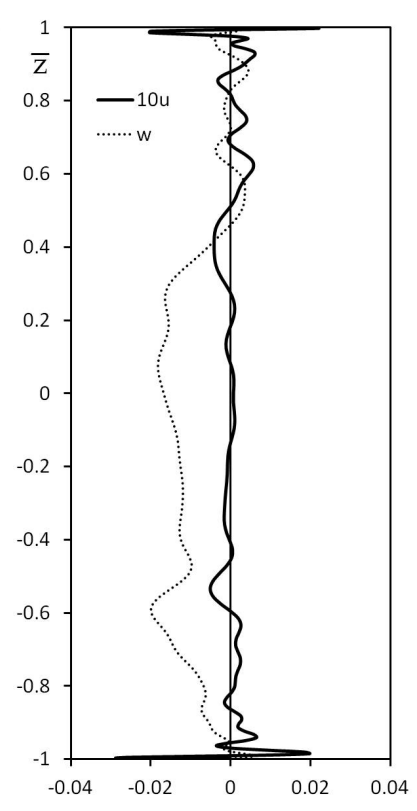

(c)

Figure 12: LES of a turbulent flow in a tall rotor-stator cavity heated from below at $R e=10^{5}$ and $R a=10^{6}$. Velocity vector field in the meridian plane showing the occurrence of small scale energetic vortices convected by the flow in the whole cavity. (b) Iso-surface of the Q-criterion showing the presence of vortical structures in the vicinity of the axis. In the meridian plane, isolines of the instantaneous temperature showing the regions of hot fluid in black and cold fluid in grey. (c) Profiles of radial, $u$, and axial, $w$, components of the mean velocity at the axis $(\bar{r}=0.0126)$. 


\section{References}

[1] G. S. Constantinescu and S. K. Lele. A highly accurate technique for the treatment of flow equations at the polar axis in cylindrical coordinates using series expansions. J. Comp. Phys., 183:165-186, 2002.

[2] R. Verzicco and P. Orlandi. A finite-difference scheme for threedimensional incompressible flows in cylindrical coordinates. J. Comp. Phys., 123:402-414, 1996.

[3] K. Mohseni and T. Colonius. Numerical treatment of polar coordinate singularities. J. Comp. Phys., 157:787-795, 2000.

[4] J. P. Boyd. Chebyshev and Fourier Spectral Methods, 2th Edition. Dover Publications, 2001.

[5] W. Huang and D. M. Sloan. Pole condition for singular problems: The pseudo-spectral approximation. J. Comp. Phys., 107:254-261, 1993.

[6] S. A. Orszag and A. T. Patera. Secondary instability of wall-bounded shear flows. J. Fluid Mech., 128:347-385, 1983.

[7] T. Matsushima and P.S. Marcus. A spectral method for polar coordinates. J. Comp. Phys., 120:365-374, 1995.

[8] Y. Zhang, A. Gandgi, A. G. Tomboulides, and S. A. Orszag. Simulation of pipe flow. Symp. on Application of Direct and Large Eddy Simulation to Transition and Turbulence, CP-551:17.1-17.9, 1994.

[9] F. Auteri and L. Quartapelle. Spectral elliptic solvers in a finite cylinder. Comm. Comp. Phys., 5 (2-4):426-441, 2009.

[10] J. Shen. Efficient spectral-Galerkin methods III: polar and cylindrical geometries. SIAM J. Sci. Comput., 18 (6):1583-1604, 1997.

[11] J. M. Lopez and J. Shen. An efficient spectral-projection method for the Navier-Stokes equations in cylindrical geometries. I. Axisymmetric cases. J. Comp. Phys., 139:308-326, 1998.

[12] J. M. Lopez, F. Marques, and J. Shen. An efficient spectral-projection method for the Navier-Stokes equations in cylindrical geometries. II. three-dimensional cases. J. Comp. Phys., 176:384-401, 2002. 
[13] P. L. O'Sullivan and K. S. Breuer. Transient growth in circular pipe flow. Part I: linear disturbances. Phys. Fluids, 6 (11):3643-3651, 1994.

[14] E. Serre and J.P. Pulicani. A three-dimensional pseudospectral method for rotating flows in a cylinder. Comput. Fluids, 30:491-519, 2001.

[15] H. Chen, Y. Su, and B. D. Shizgal. A direct spectral collocation Poisson solver in polar and cylindrical coordinates. J. Comp. Phys., 160:453-469, 2000 .

[16] E. Millour and G. Labrosse. Sensitivity of binary liquid thermal convection to confinement. Phys. Fluids, 15:2791-2802, 2003.

[17] I. Mercader, O. Batiste, and A. Alonso. An efficient spectral code for incompressible flows in cylindrical geometries. Comput. Fluids, 39:215224, 2010 .

[18] I. Raspo, S. Hugues, E. Serre, A. Randriamampianina, and P. Boutoux. A spectral projection method for the simulation of complex threedimensional rotating flows. Comput. Fluids, 31:745-767, 2002.

[19] B. Launder, S. Poncet, and E. Serre. Laminar, transitional, and turbulent flows in rotor-stator cavities. Annu. Rev. Fluid Mech., 42:229-248, 2010 .

[20] W. Heinrichs. Spectral collocation schemes on the unit disc. J. Comp. Phys., 199:66-86, 2004.

[21] R. Peyret. Spectral methods for incompressible viscous flow. Springer, 2002.

[22] S.A. Orzag and A.T. Patera. Secondary instability of wall-bounded shear flows. J. Fluid Mech., 128:347-383, 1983.

[23] H. Schlichting. Boundary layer theory. Mac Graw - Hill, New-York, 1955.

[24] M. P. Escudier. Observations of the flow produced in a cylindrical container by a rotating end wall. Exp. Fluids, 2:176-186, 1984. 
[25] F. Sotiropoulos and Y. Ventikos. Transition from bubble-type vortex breakdown to columnar vortex in a confined swirling flow. Int. J. Heat Fluid Flow, 19:446-458, 1998.

[26] E. Serre and P. Bontoux. Three-dimensional swirling flow with a precessing vortex breakdown in a rotor-stator cylinder. Phys. Fluids, 13:35003503, 2001.

[27] J. L. Stevens, J. M. Lopez, and B. J. Cantwell. Oscillatory flow states in an enclosed cylinder with a rotating endwall. J. Fluid Mech., 389:101118, 1999.

[28] E. Serre, E. Tuliszka-Sznitko, and P. Bontoux. Coupled numerical and theoretical study of the flow transition between a rotating and a stationary disk. Phys. Fluids, 6:688-706, 2004.

[29] S. Poncet, E. Serre, and P. Le Gal. Revisiting the two first instabilities of the flow in an annular rotor-stator cavity. Phys. Fluids, 21:064106, 2009 .

[30] L. Schouveiler, P. Le Gal, and M.P. Chauve. Stability of a traveling roll system in a rotating disk flow. Phys. Fluids, 10:2695-2697, 1998.

[31] J. M. Lopez and P. D. Weidman. Stability of stationary endwall boundary layers during spin-down. J. Fluid Mech., 326:373-398, 1996.

[32] O. Savas. Stability of Bödewadt flow. J. Fluid Mech., 183:77-94, 1987.

[33] G. Gauthier, P. Gondret, and M. Rabaud. Axisymmetric propagating vortices in the flow between a stationary and a rotating disk enclosed by a cylinder. J. Fluid Mech., 386:105-126, 1999.

[34] E. Séverac and E. Serre. A spectral vanishing viscosity for the LES of turbulent flows within rotating cavities. J. Comp. Phys., 226:1234-1255, 2007.

[35] E. Séverac, S. Poncet, E. Serre, and M.-P. Chauve. Large eddy simulation and measurements of turbulent rotor-stator enclosed rotor-stator flows. Phys. Fluids, 19:085113, 2007. 
[36] O. Czarny, H. Iacovides, and B. E. Launder. Precessing vortex structures in turbulent flow within rotor-stator disc cavities. Flow, Turbulence and Combustion, 69:51-61, 2002.

[37] R. J. Lingwood. Absolute instability of the Ekman layer and related rotating flows. J. Fluid Mech., 331:405-428, 1997.

[38] A. Cros, E. Floriani, P. Le Gal, and R. Lima. Transition to turbulence of the Batchelor flow in a rotor/stator device. Eur. J. Mech. B/Fluids, 24:409-424, 2005.

[39] B. Pier. Finite amplitude crossflow vortices, secondary instability and transition in the rotating-disk boundary layer. J. Fluid Mech., 487:315$343,2003$.

[40] B. Viaud, E. Serre, and J.-M. Chomaz. Transition to turbulence through steep global-modes cascade in an open rotating cavity. J. Fluid Mech., 688:493 - 506, 2011. 